\title{
Robust Normal Mode Constraints on Inner Core Anisotropy From Model Space Search
}

\author{
Caroline Beghein, ${ }^{1 *}$ Jeannot Trampert, ${ }^{1}$ \\ ${ }^{1}$ Faculty of Earth Sciences, Utrecht University, \\ Post Office Box 80021, 3508 TA utrecht, Netherlands
}

*To whom correspondence should be addressed; E-mail: beghein@geo.uu.nl

\begin{abstract}
A full model space search technique applied to measurements of anomalously split normal modes show a robust pattern of P-wave and S-wave anisotropy in the inner core. The parameter describing P-wave anisotropy changes sign around a radius of $400 \mathrm{~km}$, while $\mathrm{S}$-wave anisotropy is small in the upper twothirds of the inner core and becomes negative at greater depths. Our results agree with observed travel time anomalies of rays traveling at epicentral distances varying from $150^{\circ}$ to $180^{\circ}$. The models may be explained by progressively tilted hexagonal close-packed iron in the upper half of the inner core, but could suggest a different iron phase in the center.
\end{abstract}

The concept of inner core anisotropy is generally accepted as an explanation for both the directional dependence of PKIKP travel times and the anomalous splitting of core-sensitive free oscillations $(1,2)$. Several models have tried to explain both kinds of data, but amplitude and depth dependence of the anisotropy is still a matter of debate (1-7). In particular, models derived from the inversion of normal mode data cannot explain the large travel time anomalies observed for body waves traveling at high epicentral distances (8-10). Even joint inversions 
of normal mode and travel time data fail to reconcile all observations $(4,5,11)$. Outer core structure was even suggested to explain all existing data but couldn't account for the strong splitting of modes highly sensitive to inner core structure (6). The inner core is believed to be mainly composed of solid iron, with some unknown light elements (12-16). Although the stable phase of iron at inner core conditions is not known, mineralogical studies tend to favor a hexagonal close-packed (h.c.p.) structure. Nevertheless, the possibility of another stable phase is not excluded, especially in the presence of lighter elements (15). Estimates of the elastic properties of h.c.p. iron at high pressure and temperature (17) suggest that the basal plane of one third of the crystals would have to be aligned with Earth's spin axis to match travel time observations.

The inner core is generally modeled as a cylindrical medium with a symmetry axis parallel to Earth's rotation axis $(1,2)$. Normal mode theory $(18)$ shows that zonal structure coefficients at degrees two and four are then linearly related to three parameters that describe seismic anisotropy :

$$
c_{s 0}=\int_{0}^{R_{i c b}}\left[\alpha(r) K_{s}^{\alpha}+\beta(r) K_{s}^{\beta}+\gamma(r) K_{s}^{\gamma}\right] d r
$$

where $s$ is the spherical harmonic degree, $R_{i c b}$ stands for the radius of the inner core and $c_{s 0}$ is the measured structure coefficient at degree $s$ and order zero of a given mode. $\alpha, \beta$ and $\gamma$ are the three anisotropic parameters describing P-wave anisotropy, S-wave anisotropy and the anisotropy of waves that do not travel along the vertical or horizontal directions, respectively. They are related to the well-known Love coefficients $A, C, N, L$ and $F$ (19) as follows : $\alpha=(C-A) / A_{0}, \beta=(N-L) / A_{0}$ and $\gamma=(A-2 N-F) / A_{0}$, with $A_{0}$ the value of $A$ at the center of Earth. $C$ and $A(L$ and $N)$ are related to the speed of P-waves (S-waves) traveling parallel and perpendicular to Earth's rotation axis, respectively. $K_{s}^{\alpha}, K_{s}^{\beta}$ and $K_{s}^{\gamma}$ are the sensitivity kernels of a given mode to Earth's structure. The three model parameters were expanded on a series of five cubic spline functions with knots equally spaced through the inner 
core. The anistropic models are found by inverting or solving Eq. (1) for the spline coefficients.

We established that discrepancies among existing seismological models of inner core anisotropy are mainly due to regularization which stabilizes the inverse problem defined by Eq. (1). Regularization or damping is needed to force a solution in the presence of non-uniqueness, because of bad model sampling or contradictions in the data. Since normal mode data are not only sensitive to the core, but also to the overlying mantle, another possible source of uncertainty is the three-dimensional mantle model used to correct the measurements. From inversions by singular value decomposition (20), we found that regularization and data quality had a significant influence on the final model, while the chosen mantle model was less crucial (Fig. 1). We used different levels of damping and two sets of data, consisting of zonal degree two and degree four structure coefficients and their error estimates (21). The first data set consisted of older measurements $(22,23)$, whereas the second data set resulted from the most recent splitting measurements that followed the great Bolivia and Kuril Islands earthquakes in 1994 (24-26). All data were corrected with crustal model CRUST5.1 (27). Various mantle models (28-35) were tested (21). For all data sets, we observed that the choice of the mantle model does not have a profound effect on the solution: it affects the amplitude of the anisotropy, but not the depth pattern. The regularization, however, changes the models significantly. In general, a higher damping pushes the anisotropic signal into shallower parts of the inner core. We further observed that inversions of the older measurements produced models with the maximum of P-wave anisotropy situated at the top of the inner core, whereas including more recent data shifts this maximum to greater depths. All models obtained by damped inversions showed small amplitudes in the innermost inner core because of the nature of the sensitivity kernels. Therefore, they cannot predict the large travel time anomalies observed for waves traveling in a North-South direction (8-10).

The strong dependence of the obtained anisotropic model upon damping indicates the presence of a large model null-space (the part of the model space not constrained by the data). In 
addition, inversions do not provide realistic posterior model uncertainties, because of a complete trade-off between variance and resolution (36). To obtain all possible models of inner core anisotropy compatible with free oscillation data, we employed a forward modeling approach, the neighbourhood algorithm (NA) $(37,38)$. With such a method, the entire model space is explored, no regularization is introduced, and the model parameter uncertainties and correlations can be obtained. The NA has recently been made available and successfully used in small size tomographic studies $(29,30,39)$. We employed the NA to survey the entire model space and found the ensemble of inner core models that fit the selected splitting data (21). Because the free oscillations were best excited by the Bolivia and Kuril Islands earthquakes of 1994, we applied the NA to the most recent anomalous splitting measurements only (21). The resulting models of inner core anisotropy fit the data with a $\chi$-misfit lower than 3 , regardless of the mantle correction applied (21). The results present several robust characteristics (Fig. 2). Firstly, for most models, $\alpha$ is positive in the upper half of the inner core (radius $r=500-1200 \mathrm{~km}$ ), with amplitude increasing down to the middle of the inner core and becoming negative at greater depths. The fast direction for P-waves is thus along the rotational axis in the middle of the inner core, but becomes parallel to the equatorial plane at greater depths. Secondly, $\beta$ is small and slightly positive in the upper two-thirds of the inner core and becomes negative in the lower $400 \mathrm{~km}$ of the core. Thus, shear waves sampling the innermost inner core are expected to move faster along the rotational axis than along the equatorial plane. Thirdly, $\gamma$ is negative at the inner core boundary and undergoes two successive changes of sign around $r=1100 \mathrm{~km}$ and $r=600 \mathrm{~km}$. All these features are robust and independent of the mantle correction (21). The most interesting finding is that many models show an anisotropic signal at large depths, as opposed to inversion results where the damping drives the innermost core anisotropy to zero. There are some features that are not as well-constrained : $\alpha$ and $\gamma$ have much larger error bars at the very bottom of the inner core and their sign is not robust. Their values at large depths are 
not independently constrained by our data, as can be seen on the correlation matrix (Fig. 3).

To test the compatibility of this family of inner core models with observed differential travel time anomalies, we generated random samples, according to the posterior probablity density functions associated with each model parameter (21). P-wave velocity anomalies associated with inner core anisotropy are given by (1)

$$
\frac{\delta v}{v_{e q}}=(2 \beta-\gamma) \cos ^{2} \xi+\left(\frac{1}{2} \alpha-2 \beta+\gamma\right) \cos ^{4} \xi
$$

where $\xi$ is the angle between the ray and Earth's rotation axis and $v_{e q}$ is the equatorial velocity. Predictions of differential travel time anomalies are computed for waves traveling at about $153^{\circ}$ epicentral distance, which sample the upper $290 \mathrm{~km}$ of the inner core, and for rays traveling at $170^{\circ}$ epicentral distance, which turn at a radius of about $350 \mathrm{~km}$ (Fig. 4). We also computed predictions in the epicentral range $168^{\circ}-180^{\circ}$. All models produced, irrespective of the mantle correction, are compatible with the observed travel time anomalies of rays sampling the upper quarter of the core, and most of them predict anomalies between four and six seconds for rays traveling in a N-S direction, which confirms the estimates made from mantle corrected travel time data (10).

Our results are robust and independent of the mantle model used to correct the data. The full model space search indentified solutions previously unknown from damped inversions and produced models using normal mode data alone which agree with the observed differential travel time anomalies of rays traveling through the inner core at epicentral distances varying between $150^{\circ}$ and $180^{\circ}$. More detailed than a division between bulk and innermost inner core (11), a simple model of radially varying cylindrical anisotropy is sufficient to explain both, splitting and travel time data, without the need of outer core structure (6). A comparison with the latest determination of the elasticity of h.c.p. iron at inner core conditions (17) shows that some of our models can be explained by progressively tilted h.c.p. iron in the upper half of the inner 
core, with their symmetry axis oriented at $45^{\circ}$ from Earth's rotation axis at radius $r=900 \mathrm{~km}$ and at $90^{\circ}$ in the middle of the inner core. In the deepest inner core $(r=0-400 \mathrm{~km})$, none of our models is compatible with published data of h.c.p. iron. This might suggest the presence of another phase from these depths. Such a phase of iron could indeed be stable in the presence of impurities (15).

\section{References and Notes}

1. A. Morelli, A. Dziewonski, J. Woodhouse, Geophys. Res. Lett. 13, 1,545 (1986).

2. J. Woodhouse, D. Giardini, X.-D. Li, Geophys. Res. Lett. 13, 1549 (1986).

3. J. Tromp, Nature 366, 678 (1993).

4. J. Tromp, GSA Today. 5, 137 (1995).

5. J. Durek, B. Romanowicz, Geophys. J. Int. 139, 599 (1999).

6. B. Romanowicz, L. Bréger, J. Geophys. Res. 105, 21,559 (2000).

7. K. Creager, Earth's Deep Interior: Mineral Physics and Tomography From the Atomic to the Global Scale, Seismology and Mineral Physics, Geophys. Monogr. Ser., S. Karato, ed. (AGU, Washington, D.C., 2000), vol. 117, pp. 63-87.

8. W.-J. Su, R. Woodward, A. Dziewonski, J. Geophys. Res. 100, 9,831 (1995).

9. X. Song, J. Geophys. Res. 101, 16,089 (1996).

10. X. Sun, X. Song, Earth planet. Sci. Lett. 199, 429 (2002).

11. M. Ishii, A. Dziewonski, Proc. Natl. Acad. Sci. USA 99, 14026 (2002). 
12. F. Birch, J. Geophys. Res. 69, 4,377 (1964).

13. L. Stixrude, E. Wasserman, R. Cohen, J. Geophys. Res. 102, 24,729 (1997).

14. H. Mao, et al., Science 292, 914 (2001).

15. J. Lin, D. L. Heinz, A. Campbell, J. Devine, G. Shen, Science 295, 313 (2002).

16. C. Gessman, B. Wood, Earth planet. Sci. Lett. 200, 63 (2002).

17. G. Steinle-Neumann, L. Stixrude, R. Cohen, O. Gülseren, Nature 413, 57 (2001).

18. J. Tromp, Geophys. J. Int. 121, 963 (1995).

19. A. Love, A Treatise on the Theory of Elasticity (Cambridge Univ. Press, 1927).

20. M. Matsu'ura, N. Hirata, J. Phys. Earth 30, 451 (1982).

21. Materials and methods are available as supporting material on Science Online.

22. M. Ritzwoller, G. Masters, F. Gilbert, J. Geophys. Res. 93, 6,369 (1988).

23. R. Widmer, G. Masters, F. Gilbert, Geophys. J. Int. 111, 559 (1992).

24. J. Tromp, E. Zanzerkia, Geophys. Res. Lett. 22, 2,297 (1995).

25. X. He, J. Tromp, J. Geophys. Res. 101, 20,053 (1996).

26. J. Resovsky, M. Ritzwoller, J. Geophys. Res. 103, 783 (1998).

27. W. Mooney, G. Laske, G. Masters, J. Geophys. Res. 103, 727 (1998).

28. J. Resovsky, M. Ritzwoller, J. Geophys. Res. 104, 993 (1999).

29. J. Resovsky, T. Trampert, Nature (2002). Submitted. 
30. C. Beghein, J. Resovsky, J. Trampert, Geophys. J. Int. 149, 646 (2002).

31. G. Masters, S. Johnson, G. Laske, H. Bolton, Phil. Trans. R. Soc. London 354, 1,385 (1996).

32. J. Ritsema, H.-J. van Heijst, Science 83, 243 (2000).

33. X. Li, B. Romanovwicz, J. Geophys. Res. 101, 22,245 (1996).

34. G. Masters, G. Laske, H. Bolton, A. Dziewonski, Earth's Deep Interior: Mineral Physics and Tomography From the Atomic to the Global Scale, Seismology and Mineral Physics, Geophys. Monogr. Ser, S. Karato, ed. (AGU, Washington, D.C., 2000), vol. 117, pp. 63-87.

35. W.-J. Su, R. Woodward, A. Dziewonski, J. Geophys. Res. 99, 6,945 (1994).

36. G. Backus, F. Gilbert, Phil. Trans. R. Soc. London A266, 123 (1970).

37. M. Sambridge, Geophys. J. Int. 138, 479 (1999).

38. M. Sambridge, Geophys. J. Int. 138, 727 (1999).

39. J. Resovsky, T. Trampert, Geophys. J. Int. 150, 665 (2002).

40. M. Sambridge generously provided the codes of the neighbourhood algorithm. We wish to thank K.C. Creager who provided the $\mathrm{PKP}(\mathrm{BC})-\mathrm{PKP}((\mathrm{DF})$ and $\mathrm{PKP}(\mathrm{AB})-\mathrm{PKP}((\mathrm{DF})$ data and the code giving travel time anomalies as a function of the ray angle, and X. Song who sent us the differential travel time data for the epicentral distance range $168^{\circ}-180^{\circ}$. We are grateful to $\mathrm{H}$. Paulssen for the numerous discussions concerning body-waves and for computing the reference travel times for P-waves inside the inner core. We thank J. Resovsky for his advice on the normal mode data. We also appreciated the discussion with M. Ishii concerning her work on the inner core. Finally, we wish to thank all people who made their normal mode measurements and mantle models available in various publications. 


\section{Supporting Online Material}

www.sciencemag.org

1 file containing Methods (5 notes) 
Fig. 1. Models resulting from the inversion of older data are shown in panels (A) and (B), those resulting from the inversion of recent data are shown in panels (C) and (D). Two levels of damping were applied. The upper panels correspond to highly damped models, the lower panels are models for which the constraint of the damping was lower. The solid lines represent P-wave anisotropy, the dotted lines S-wave anisotropy and the dashed lines parameter $\gamma$. Different mantle models (21) were used to correct the data, yielding the inner core models in color and in the grey areas.

Fig. 2. Models resulting from the application of the neighbourhood algorithm, using mantle (29). The thin dotted line represents the mean model and the thick surrounding lines correspond to two standard deviations taken from the posterior probability density functions obtained from the NA. $A_{0}$ is the value of elastic parameter $A$ at the center of the Earth.

Fig. 3. Correlation matrix corresponding to the family of models in Fig. (2). Indices correspond to the radius of the different spline knots. The off-diagonal elements in the matrix describe how the data link the different model parameters.

Fig. 4. Predictions of differential travel time anomalies $\operatorname{PKP}(\mathrm{BC})-\mathrm{PKP}(\mathrm{DF})$ at epicentral distances of $153^{\circ}(\mathrm{A})$ and $\mathrm{PKP}(\mathrm{AB})-\mathrm{PKP}(\mathrm{DF})$ at $170^{\circ}(\mathrm{B})$ and in the range $168^{\circ}-180^{\circ}(\mathrm{C})$. $\xi$ is the angle between Earth's rotation axis and the direction of propagation of the wave. The dots in the upper and lower panels are data points for waves travelling in the epicentral distance range $147^{\circ}-153^{\circ}$ and $167^{\circ}-173^{\circ}$, respectively (7). The diamonds of the third panel are binned AB-DF data between 168 and $180^{\circ}$, with two standard deviations (10). These estimations are based on random prediction from the family of models shown in Fig. 2. The solid vertical lines represent two standard deviations of our predictions (21). 


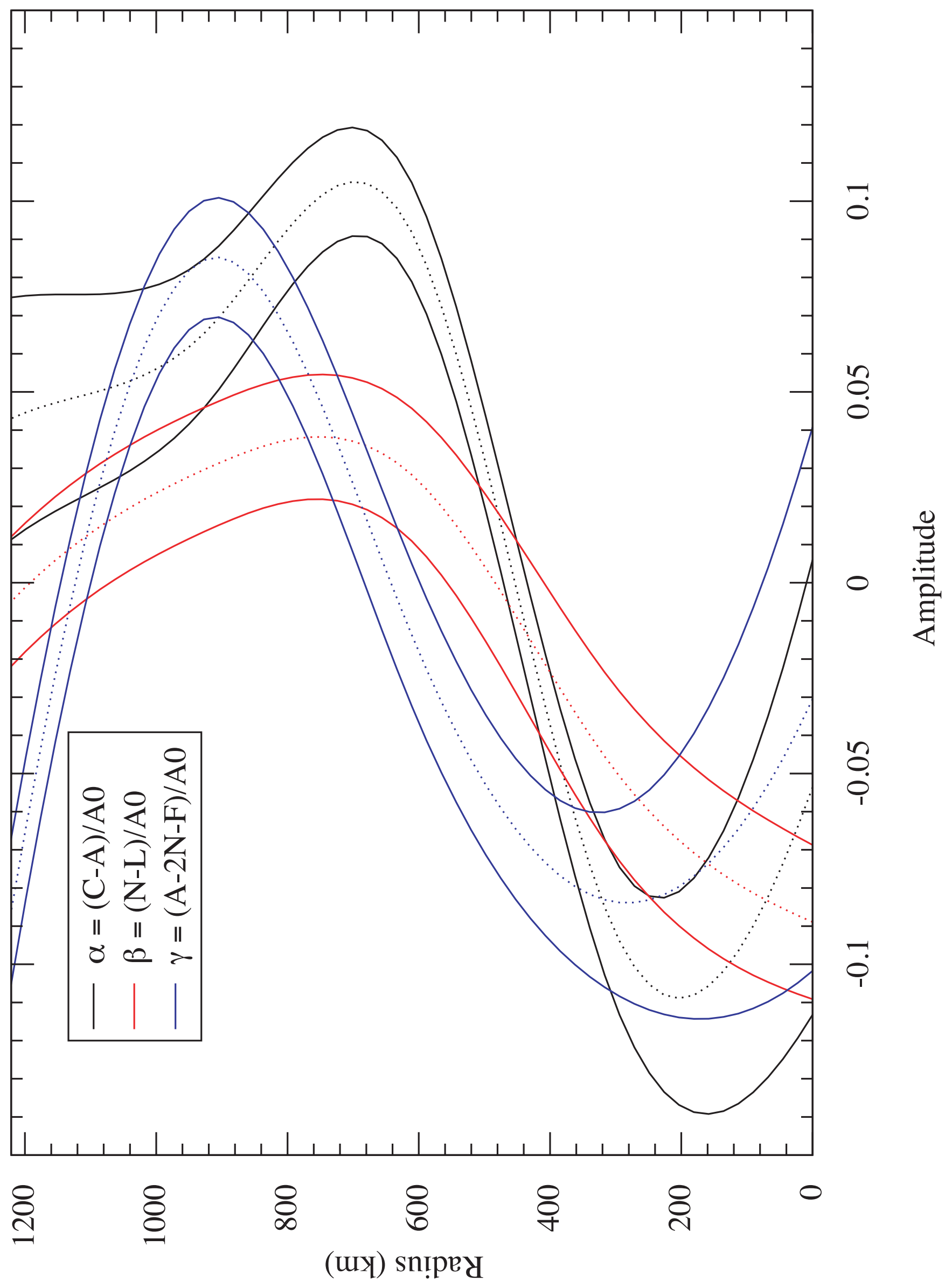




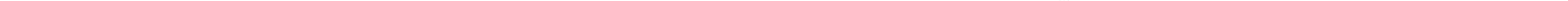



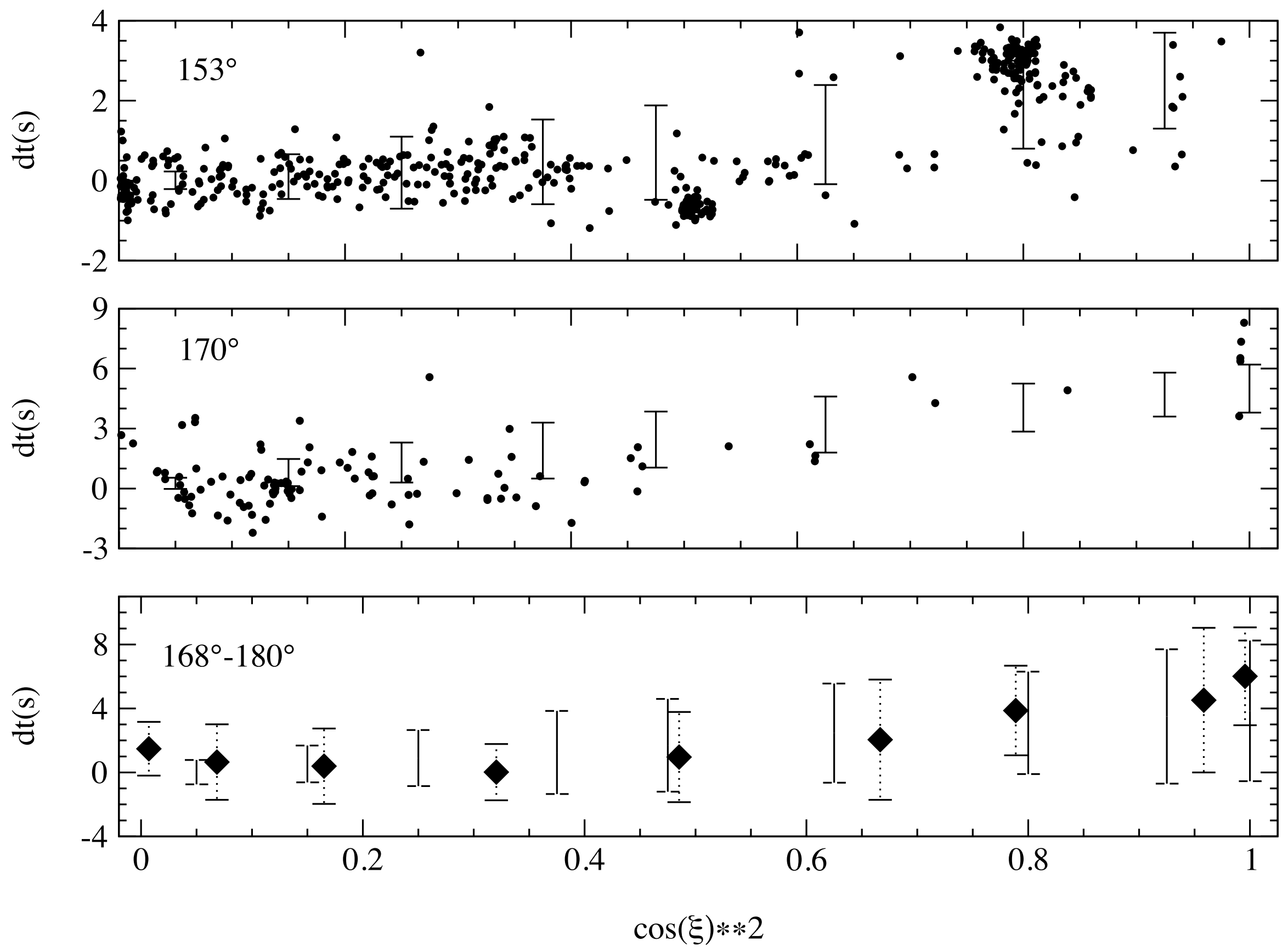